\title{
The Need for Command and Control Instant Message Adaptive Interfaces: Lessons Learned from Tactical Tomahawk Human-in-the-Loop Simulations
}

\author{
M.L. Cummings \\ Massachusetts Institute of Technology \\ MissyC@mit.edu
}

\begin{abstract}
In the recent development of a human-in-the-loop simulation test bed designed to examine human performance issues for supervisory control of the Navy's new Tactical Tomahawk missile, measurements of operator situation awareness (SA) and workload through secondary tasking were taken through an embedded instant messaging program. Instant message interfaces (otherwise known as "chat"), already a means of communication between Navy ships, allow researchers to query users in real-time in a natural, ecologic setting, and thus provide more realistic and unobtrusive measurements. However, in the course of this testing, results revealed that some subjects fixated on the real-time instant messaging secondary task instead of the primary task of missile control, leading to the overall degradation of mission performance as well as a loss of SA. While this research effort was the first to quantify command and control performance degradation as a result of instant messaging, the military has recognized that in its network centric warfare quest, instant messaging is a critical informal communication tool, but has associated problems. Recently a military spokesman said that managing chat in current military operations was sometimes a "nightmare" because military personnel have difficulty in handling large amounts of information through chat, and then synthesizing knowledge from this information. This research highlights the need for further investigation of the role of instant messaging interfaces both on task performance and situation awareness, and specifies how the associated problems could be ameliorated through adaptive display design.
\end{abstract}

\section{Introduction}

It has long been recognized that humans and computers/machines possess unique strengths and weaknesses in supervisory control domains (Chapanis et al., 1951). However, current trends in human-machine research and design indicate that instead of a mutually exclusive assignment of tasks and functions, the more dynamic approach of adaptive automation can leverage the strengths of humans and computers to improve overall system performance while mitigating the negative aspects of both (see Scerbo, 1996 and Kaber et al., 2001.) The use of flexible and adaptive automation in supervisory control has been shown to promote improved automation monitoring (Parasuraman et al., 1996) and superior task performance (Hilburn et al., 1997; Scallen \& Hancock, 2001), as well as improved situation awareness in complex system management (Kaber \& Endsley, 2004). Military agencies, both American and European, have invested significant resources in adaptive automation and intelligent decision support research for pilots, yielding systems such as the Cognitive Cockpit (Taylor et al., 2002; Taylor, Howells, \& Watson, 2000) and the Rotorcraft Pilot's Associate (Miller, Hannen, \& Guerlain, 1999). 
In addition, adaptive multisensory interfaces have been investigated for aircraft navigation, visual target acquisition, and adaptive spatial audio displays for aircrew (Haas et al., 2001). Recent research in adaptive human-computer interaction has extended into the unmanned aerial vehicle control domain with adaptive aiding through psychophysiology measures in the location and designation of targets for Unmanned Combat Aerial Vehicles (UCAVs) (Wilson \& Russell, 2004).

Typical focus of these and other similar adaptive automation and intelligent aiding projects has been to support the primary psychomotor and cognitive tasks of pilots and controllers, i.e. flying the aircraft, acquiring a target etc. However, recent research examining human supervisory control issues for in-flight control of the U.S. Navy's Tactical Tomahawk missile revealed the need for adaptive automation research in the area of secondary tasking, specifically in the management of datalink communications manifested through instant messaging interfaces. In general, research surrounding adaptive automation has primarily focused on application to primary tasks but it could be that focusing efforts on designing adaptive interfaces for secondary tasking may mitigate the need for primary tasking adaptive automation. Kaber \& Riley (1999) demonstrated that secondary tasking measures can provide appropriate cues for primary tasking adaptive allocation, but the impact of secondary tasking adaptive automation on a primary task has not been addressed.

The need for an intelligent manager of secondary tasking such as instant messaging is not limited to just the domain of in-flight missile control, as instant messaging was a primary means of communication between Navy ships during Operation Iraqi Freedom in 2003. While instant messaging, otherwise known as chat, has many advantages for rapid response in critical time-pressure command and control situations, operational commanders have found it difficult to handle large amounts of information generated through chat, and then synthesize relevant knowledge from this information (Caterinicchia, 2003). This paper will discuss the first attempt to quantify human supervisory command and control performance degradation as a result of interference from instant messaging secondary tasking, and then discuss how these results motivate the need for adaptive automation for datalink communications management.

\section{Background}

The Tomahawk missile can be fired from over 1000 miles away from its intended target with an accuracy of meters. Previous versions of the missile were "fire-and-forget" in that once launched, their courses of action could not be modified. The newest version, the Tactical Tomahawk, will have the capability of redirection in-flight through transmission of GPS data. This new capability of retargeting missiles in flight represents a major shift not only in the human supervisory control issues for the Tomahawk missile, but also how a dynamic system that requires constant human replanning efforts could and should be designed.

Figure 1 represents a dual screen interface that was developed to simulate the human supervisory control environment of a Tactical Tomahawk controller whose primary tasking is to monitor the progress of the missiles in flight and redirect them as required by changes in the combat environment. The monitor display on the right, otherwise known as the map display, represents all missiles and targets in a geo-spatial 


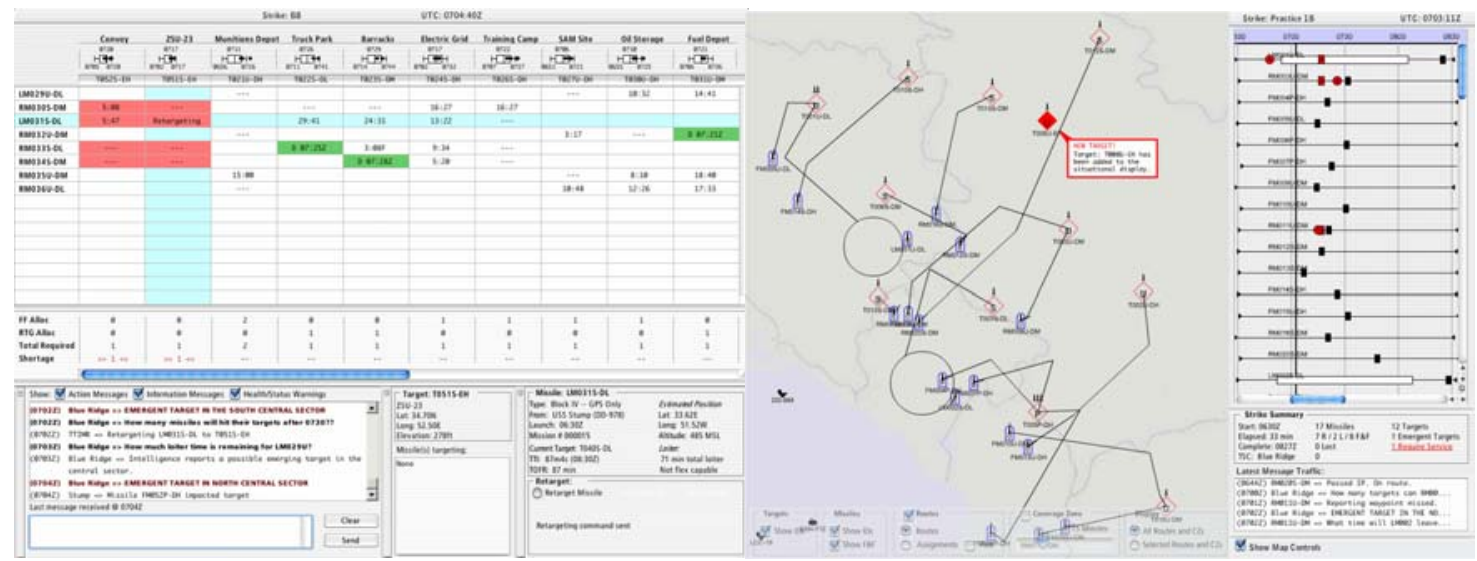

Figure 1: The Tactical Tomahawk Interface for Monitoring and Retargeting

display superimposed on a map of the local terrain. The map allows the controller to perceive missile information geographically, while an associated time bar allows the controller to perceive important temporal missile relationships such as launch time, time of impact, and time of fuel remaining, all in comparison to the actual time and each of the other missiles. The left side of the display is designed to support the real-time retargeting decision for missiles as well as related communication activities. This display contains the primary decision support tool, known as the decision matrix as well as an instant messaging interface, known as the "chat box". The decision matrix provides the user with the ability to see not only the current status of all missiles currently capable of retargeting, but all future possibilities as well. (See Cummings, 2003 for a more detailed description of the design.)

The chat box on the left display, enlarged in Figure 2, allows controllers to receive instant messages that contain basic status information as well as instructions for action or queries for information. Three different types of messages come in through the chat box, which can be filtered at will by the operator: information messages, health and status messages, and action messages. Information messages are those that deliver noncritical information from either a human or automated agent. Health and status messages are messages from the missiles, updating the controller on position and system status. The last and most important category of incoming instant messages are the action messages, which require the operator to either take some action or communicate back some piece of information to a superior.

In the course of human-in-the-loop experiments with this test bed, two performance measures were taken through this instant messaging interface: 1) secondary tasking as a measure of workload, and 2) situation awareness. Secondary tasking is a commonly used workload measurement tool that requires a subject, assigned a primary task, to use any spare mental capacity to attend to a secondary task. Measuring workload through primary tasking and other aggregate measures like operator utilization are important, but the use of secondary task measurements provides a more comprehensive workload analysis (Wickens \& Hollands, 2000). Because traditional secondary tasking such as tapping and time estimation tasks can be intrusive and introduce an unrealistic artifact during testing (Williges \& Wierwille, 1979), the instant messaging interface was used as an embedded measurement tool to counter this confound. Embedded secondary 


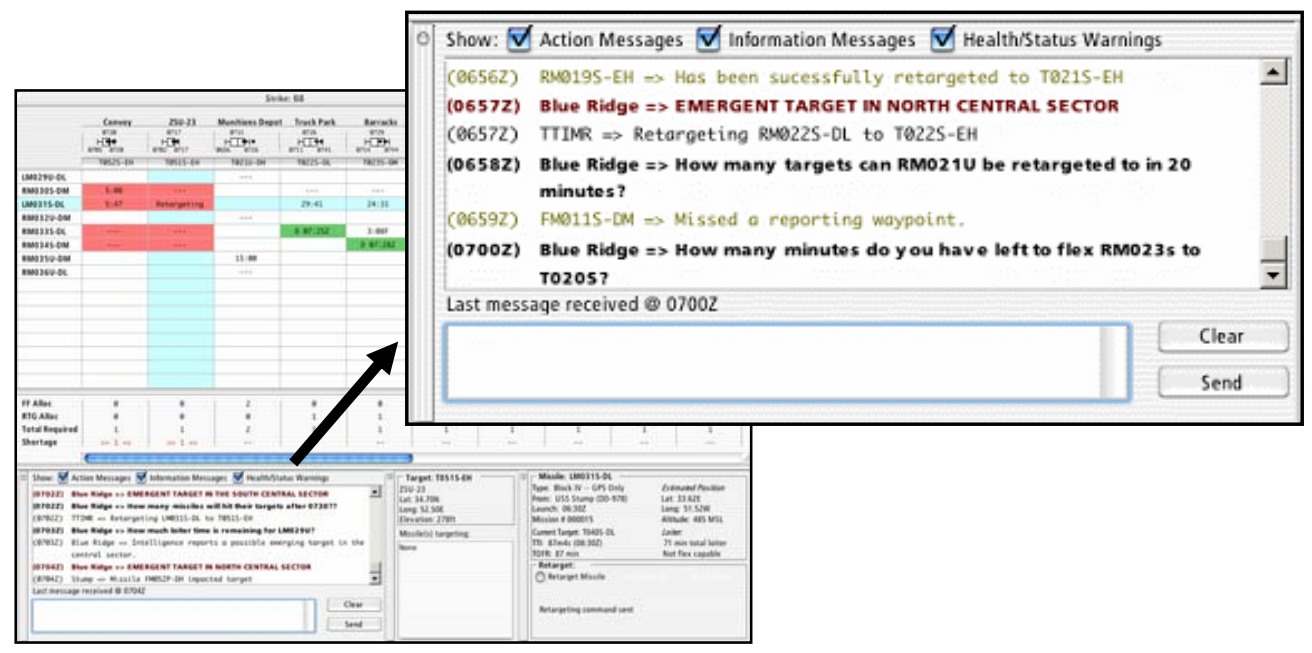

Figure 2: The TTIMR Chat Box

tasks do not fundamentally change the task or task performance and provide more sensitive measurements because of their unobtrusiveness in a natural, ecologic setting (Shingledecker, 1987; Tsang \& Wilson, 1997; Wickens \& Hollands, 2000). The chat box represents current technology in place on naval vessels, and is a natural embedded measurement tool since responding to communications in this format is familiar to Navy personnel. In addition, an important experimental design consideration for human subject testing is external validity, which is a measure of how well experimental results will generalize to an operational setting. One way to strengthen external validity is to represent the operational environment as accurately as possible in an experimental setting (Adelman, 1991).

The second performance measure taken through the embedded instant messaging interface was situation awareness. Situational awareness (SA) is generally defined as having three levels, which are: 1) the perception of the elements in the environment, 2) the comprehension of the current situation, and 3) the projection of future status (Endsley, 1988; Endsley, 1995). While SA can decrease under high workload due to competition for attentional resources (Wickens, 1995), it can also decrease under low workload due to boredom and complacency (Rodgers, Mogford, \& Strauch, 2000). Situation awareness has long been recognized as a critical human factor in military command and control systems. Military command and control centers must attempt to assimilate and reconstruct the battle picture based on information from a variety of sensor sources such as weapons, satellites, and voice communications. In the human supervisory control domain of remotely piloted vehicles, which is similar to the Tactical Tomahawk domain, poorly designed interfaces have led to reduced situation awareness as well as degraded mission performance (Ruff, Narayanan, \& Draper, 2002). Because of the complexity and dynamic nature of the command and control environment, maintenance of situational awareness is considered to be of utmost importance (Klein, 2000; Santoro \& Amerson, 1998). 


\section{Experimental Results}

A series of human-in-the-loop experiments using the interface in Figure 1was conducted using 42 Navy subjects to evaluate a number of performance measures, which included the secondary workload and situation awareness metrics. The embedded instant messaging interface queried users during both monitoring periods, which represented intervals of low workload, and also during periods of high workload in which operators were required to retarget a missile based on pre-specified rules of engagement and orders from superiors. These queries introduced through the chat interface were action messages that required the subject to communicate to the supervisor a piece of information. The following are samples of typical queries subjects were asked through the embedded interface:

- What is the time to impact for the last missile striking a particular target?

- How many missiles will have hit their targets by 0730Z?

- How many targets are not currently receiving their required number of missiles?

- If you needed to get a missile to a particular target ASAP, what missile would it be?

- How many targets have been destroyed?

Secondary tasking was measured as the time it took subjects to respond to the questions and SA was measured based on the correctness of the answer. Secondary tasking analysis (detailed in Cummings \& Guerlain, 2004) demonstrated that during retargeting scenarios, workload increased for the subjects as compared to workload during monitoring situations. In addition, as the complexity of the scenarios increased and as number of missiles available for control was increased, the secondary tasking measurements demonstrated higher workload and increasing overall performance degradation.

The answers to the SA questions for each test session provided an overall SA measurement, and were analyzed through a linear mixed model. The results, reported elsewhere (Cummings \& Guerlain, in review) revealed no significant differences in situation awareness for varying workload levels. The lack of a significant SA effect indicates that perhaps the measurement technique was ineffectual and that the questions and timing should be examined more closely to ensure they are sensitive enough to detect changes in SA. However, as will be detailed in the next section, it is also possible that despite the fact the embedded instant messaging interface was designed to unobtrusively gather SA data, its existence could confound the data by causing some subjects to lose SA.

\section{Unexpected Results}

During the conduct of the experiment and subsequent data analysis, an unexpected behavioral trend was noted in regards to the use of the instant message interface as the primary means of communication and as an embedded measurement tool. Many subjects fixated on the instant messaging and ignored primary tasking of retargeting missiles in urgent situations. This occurred despite the fact that all subjects were repeatedly instructed that retargeting situations were their primary priority tasking 
and that answering queries through the chat box was the least important of all tasks. Despite this heavy training emphasis on only attending to the chat box when nothing else was happening, many subjects fixated on the communications and would answer all queries before attending to the more pressing retargeting problems. Because operators must time-share attention between the monitoring task and the execution of any required actions, the addition of instant messages could be costly from an operational perspective both in terms of potential human error and overall attainment of goals.

To investigate what "cost" could be associated with this instant messaging fixation, the impact of over-attention to chat was explored statistically in the experimental data to assess the impact on the subjects' overall performance. To statistically confirm any relationship for instant message fixation (termed chatty) on overall performance, a correlation was examined between the chatty factor and an overall objective performance score based on decision time and decision accuracy. A person was categorized as chatty if they continued responding to an action communication message ten seconds after notification of a retargeting situation. This comparison revealed a moderate correlation, Pearson correlation $=-.292, \mathrm{p}=.008(\alpha=.05)$, so the overall performance suffered for those subjects who ignored primary tasking in favor of chat.

While correlations can give some insight into a relationship between variables, they do not establish cause and effect. Given that the correlation showed that it was possible that instant messaging fixation could be a significant predictor, multiple linear regression was used to determine if the chatty variable would be a predictor for overall performance in addition to other primary independent variables. The chatty factor was significant, $(\beta=-.218, p=.015, \alpha=.05)$. These results can be interpreted as an indication that those people who fixated on instant message management experienced overall lower performance scores than those who did not engage in this behavior. This analysis can be considered exploratory only since this was a supplementary analysis and not originally part of the intended experimental design, but it does suggest that much more research needs to be completed in the impact of instant messaging on task performance.

\section{Discussion}

There are obvious advantages to an informal real-time communications network embodied in instant messaging, such as rapid response to inquiries, the ability to communicate with multiple people at once, as well as the ability to access the archived chat session for clarification or as a historical record. However, there are also many potential drawbacks. Previous research has demonstrated that chat can be disruptive, and the flow of conversation can be awkward since there is no non-verbal feedback like what would occur between two people in a face-to-face conversation (Tang, Yankelovich, \& Begole, 2000). In time-pressure scenarios, interruptions of a primary task caused by an interruption mechanism like chat can increase mental processing time and induce errors in the primary task (Cellier \& Eyrolle, 1992). In the air traffic control (ATC) domain, communication between pilots and controllers through datalink has been explored, and in general causes higher workloads. Because of the problems with overloading and situation awareness in the primary tasking of controlling aircraft, ATC datalink is thought to be useful only in non-critical operations (Harris and Lamoureaux, 2000). 
In a supervisory control tasks such as command and control, air traffic control, or the monitoring of process control displays, operators spend time monitoring unfolding events, which may or may not be changing rapidly. In addition, they also will periodically engage in interactive control tasks such as giving aircraft instructions or raising a fluid level in a tank. When task engagement occurs, operators must both concentrate attention on the primary task, but also be prepared for alerts for external events. This need to concentrate on a task, yet maintain a level of attention for alerts causes operators to have a conflict in mental information processing. Concentration on a task requires task-driven processing which is likely to cause decreased sensitivity or attention to external events. Interrupt-driven processing, needed for monitoring alerts, occurs when people are sensitized to possible problems and expect distraction.

While interrupt and task driven processing can both be present in a person, attention must be shared between the two and switching can incur cognitive costs that can potentially result in errors (Miyata \& Norman, 1986). The conflict between focusing on tasks and switching attention to interruptions is a fundamental problem for operators attempting to supervise a complex system which requires dedicated attention but also requires operators to respond to an instant messaging interface, which represents interruptions. In addition, Gopher et al. (1996) demonstrated that not only is there a measurable cost in response time and decision accuracy when switching attention between tasks, but costs are also incurred by the mere reconsideration of switching tasks. Monitoring instant messaging while attending to a primary task causes people to divert their attention, and even if the attention division is only momentary, this reconsideration incurs a cost. While users in office environments pay a cognitive cost in switching between task and interruption processing, for people interacting with complex supervisory systems such as process control or air traffic management, the cost for potential errors can be much higher and potentially catastrophic. For example, in 1987, a Northwest airline crew was interrupted in the middle of a pre-taxi checklist when contacted by ATC with an updated clearance. The crew forgot to complete the checklist and attempted a take off with an incorrect flap setting, resulting in a crash that killed 154 passengers and crew (NTSB, 1988)

It has been well established that interruptions can be disruptive to computer tasks (Cutrell, \& Horvitz, 2001, 2000a, 2000b; Gillie \& Broadbent, 1989). Relevant to human supervisory control tasks under time pressure, computer-initiated interruptions cause a significant increase in primary task completion times (Bailey, Konstan, \& Carlis, 2000, 2001). In recent instant message research, Czerwinski, Cutrell and Horvitz (2000b) demonstrated that instant messages delivered during the evaluation of a list of computergenerated results were more disruptive than messages delivered in other stages of the task. Furthermore, instant messaging has been demonstrated to negatively impact faster, stimulus-driven search tasks more than slower search tasks (Cutrell et al., 2001; Czerwinski et al., 2000b). Addressing possible instant messaging management strategies, Czerwinski, et al., (2000b) demonstrated that withholding instant messages until key tasks are detected can reduce the disruptive effects of instant messages. In addition, they determined that instant messages that were relevant to current tasking were less disruptive than those deemed irrelevant. This finding begs the question, "Is it possible to develop an intelligent automated agent that can aid the operator both in sorting between 
relevant and non-relevant messages as well as determining priority scheduling that interrupts the user during low cognitive loads?"

Because supervisory control operators must time-share attention between monitoring tasks and the execution of any required actions, the addition of an instant messaging system as a secondary task will require that attention be further divided. If the power of an intelligent automated agent was harnessed so that the computer could determine more optimal scheduling patterns for the presentation of instant messages, it is possible that both errors could be mitigated and overall performance improved. However, the design of adaptive systems is difficult and highly complex (see Kaber et al., 2001 for a review), and it is possible that increasing levels of automation can cause a new set of problems (Endsley \& Kaber, 1999; Parasuraman \& Riley, 1997; Parasuraman, Sheridan, \& Wickens, 2000). Unfortunately, instant messaging interfaces and possible cognitive consequences is a research area that has received limited attention (Olson \& Olson, 2003), so how to design an effective adaptive interface for instant message management is not obvious.

\section{Designing to Minimize Interruptions}

Norman (1986) contends that there seven stages of user activity which are the establishment of a goal, forming of an intention, specifying an action, executing this action and then evaluation of this action which includes perception, interpretation, and evaluation of result in comparison to expectation. This model suggests that the most appropriate time and place for interruption would be after the final evaluation of expectation and the forming of a new goal. Bailey et al. (2000) confirmed this assertion experimentally and recommended that computer-initiated interruptions should occur when a user reaches a "task boundary" or during a period of low interaction. In a supervisory task, the ideal interruption period would translate to the time when an operator was merely monitoring a system and not engaged in any decision-making or system evaluation. Due to the dynamic nature of complex systems, however, interruptions may not always be scheduled in these times.

According to Norman's seven stages of user activity, interruptions incur the greatest cognitive costs during the planning stages (forming an intention and developing an action) as well as the evaluation stages (interpretation and expectation evaluation) (Miyata \& Norman, 1986). Czerwinski, Cutrell, \& Horvitz (2000b) demonstrated that for desktop tasks, instant messaging disrupts the execution and evaluation stages. Because the stages where interruptions would be most disruptive are cognitive activities that are not generally obvious to the computer, it is difficult for a computer to anticipate the stage of user activity and interrupt in periods that do not incur high cognitive costs. To effectively address the interruption problem in instant messaging, one challenge then is to develop an adaptive system in which the computer schedules interruptions to occur in periods of low interaction, which under Norman's model would occur after the conclusion of evaluation and before a new goal is formed. 


\section{Conclusion}

Instant messaging can be a powerful distributed communications tool, but as has been demonstrated in the Tactical Tomahawk human-in-the-loop research, as a secondary task, it can disrupt primary tasking and cause overall human performance degradation. This problem could be ameliorated by the use of adaptive automation. Adaptive interfaces that intelligently manage incoming messages could provide lower workloads and less interruptions for operators, however it is not clear what negative consequences adaptive automation might cause. An adaptive instant messaging interface will introduce added complexity into a system, both from a technical and human reasoning perspective. Even though workload could be lowered by automated mediation, a loss of situation awareness could result since not all messages deemed significant would be seen by the controller. Seemingly irrelevant messages may be withheld from the controller, which although not critical, would have added to the controller's overall SA. For example, a conversation between two ships in a battlegroup about minor weapons malfunctions on one of the ships might not seem to be important to a controller on a third ship, and the conversation suppressed as a result to the third controller. However, if a major system failure was experienced by the third ship and weapons control needed to be passed to one of the other two platforms, a relatively straightforward decision would be complicated by a general lack of understanding of the developing situation within the battlegroup.

Because of the cognitive complexities introduced by adaptive automation, specific areas for the investigation of the use of adaptive interfaces in instant message management should include:

- As an intelligent agent mediated instant message viewing, how would the operator's knowledge states and decision processes be affected?

- When should an adaptive chat management tool interrupt the operator and under what conditions?

- Is there a principled way for this tool to infer the operators' workload and ability to cognitively attend to communication messages?

- How will an adaptive chat management strategy affect overall human performance, situation awareness, and frustration?

Instant messaging is predicted to grow in the workplace more than $150 \%$ in the next few years (Thorsberg, 2003), thus the problems with primary task disruption will also become evident in many more domains. Despite possible drawbacks, the use of instant messaging to communicate and manage real-time, dynamic problems will likely extend beyond social, business, and military command and control systems in the future. With improvements in communications technology, the use of near-synchronous text messaging for complex human supervisory control could become useful in domains such as commercial aviation, ground transportation systems, and any realm in which the environment limits voice communications such as high-noise settings and remote operations. However, given the complex sociotechnical natures of these domains and the propensity for instant message distraction, more research is needed in the development of adaptive communication management. 


\section{Acknowledgments}

This material is based in part upon work supported by the Naval Surface Warfare Center, Dahlgren Division, through a grant from the Office of Naval Research Knowledge and Superiority Future Naval Capability Program. Any opinions, findings, and conclusions or recommendations expressed in this material are those of the authors and do not necessarily reflect the views of NSWC/DD or ONR.

\section{References}

Adelman, L. (1991). Experiments, Quasi-Experiments, and Case Studies: A Review of Empirical Methods for Evaluating Decision Support Systems. IEEE Transactions on Systems, Man, and Cybernetics, 22(2), 293-301.

Bailey, B. P., Konstan, J. A., \& Carlis, J. V. (2000). Measuring the Effects of Interruptions on Task Performance in the User interface. Paper presented at the IEEE Systems, Man, and Cybernetics, Nashville, TN.

Bailey, B. P., Konstan, J. A., \& Carlis, J. V. (2001). The Effects of Interruptions on Task Performance, Annoyance, and Anxiety in the User interface. Paper presented at the Interact 2001, Tokyo.

Caterinicchia, D. (2003, June 23). DOD chat use exploded in Iraq. Federal Computer Week.

Cellier, J., \& Eyrolle, H. (1992). Interference between switched tasks. Ergonomics, 35, 25-36.

Chapanis, A., Frick, F. C., Garner, W. R., Gebhard, J. W., Grether, W. F., Henneman, R. H., Kappaif, W. E., Newman, E. B., \& Williams, A. C. (1951). Human Engineering for an effective air navigation and traffic control system. In P. M. Fitts (Ed.). Washington DC: National Research Council.

Cummings, M. (2003). Designing Decision Support Systems for Revolutionary Command and Control Domains. University of Virginia, Charlottesville.

Cummings, M. L., \& Guerlain, S. (2004). Using a Chat Interface as an Embedded Secondary Tasking Tool. Paper presented at the Human Performance, Situation Awareness and Automation Technology II Conference, Daytona Beach, FL.

Cummings, M.L., and Guerlain, S., in review, Developing Operator Capacity Estimates for Supervisory Control of Autonomous Vehicles, Human Factors.

Cutrell, E., Czerwinski, M., \& Horvitz, E. (2001). Notification, Disruption, and Memory: Effects of Messaging Interruptions on Memory and Performance. Paper presented at the Interact 2001, Tokyo. 
Czerwinski, M., Cutrell, E., \& Horvitz, E. (2000a). Instant Messaging and Interruption: Influence of Task Type on Performance. Paper presented at the OZCHI 2000, Sydney, Australia.

Czerwinski, M., Cutrell, E., \& Horvitz, E. (2000b). Instant messaging: Effects of relevance and time. Paper presented at the People and Computers XIV: HCI 2000.

Endsley, M. (1988). Design and Evaluation for situation awareness enhancement. Paper presented at the Human Factors Society 32nd Annual Meeting.

Endsley, M. (1995). Measurement of situation awareness in dynamic systems. Human Factors, 37(1), 65-84.

Endsley, M., \& Kaber, D. B. (1999). Level of automation effects on performance, situation awareness and workload in a dynamic control task. Ergonomics, 42(3), 462-492.

Gillie, T., \& Broadbent, D. (1989). What makes interruptions disruptive? A study of length, similarity, and complexity. Psychological Research, 50, 243-250.

Gopher, D., Greenshpan, Y., \& Armony, L. (1996). Switching attention between tasks: Exploration of the components of executive control and their development with training. Paper presented at the Human Factors and Ergonomics Society 40th Annual Meeting, Philadelphia, PA.

Haas, M. W., Nelson, W. T., Repperger, D., Bolia, R., \& Zacharias, G. (2001). Applying Adaptive Control and Display Characteristics to Future Air Force Crew Stations. International Journal of Aviation Psychology, 11(2), 223-235.

Harris, S., \& Lamoureaux, T. (2000). The future implementation of datalink technology: The controller-pilot perspective. In P. T. McCabe \& M. A. Hanson \& S. A. Robertson (Eds.), Contemporary Ergonomics 2000 (pp. 7-11). London: Taylor and Francis.

Hilburn, B., Jorna, P. G., Byrne, E. A., \& Parasuraman, R. (1997). The effect of adaptive air traffic control (ATC) decision aiding on controller mental workload. In M. Mouloua \& J. Koonce (Eds.), Human-automation interaction: Research and practice (pp. 84-91). Mahwah, NJ: Lawrence Erlbaum.

Kaber, D. B., \& Riley, J. M. (1999). Adaptive Automation of a dynamic control task based on secondary task workload measurement. International Journal of Cognitive Ergonomics, 3, 169-187. 
Kaber, D. B., Riley, J. M., Tan, K., \& Endsley, M. R. (2001). On the Design of Adaptive Automation for Complex Systems. International Journal of Cognitive Ergonomics, 5(1), 37-57.

Kaber, D. B., \& Endsley, M. R. (2004). The effects of level of automation and adaptive automation on human performance, situation awareness and workload in a dynamic control task. Theoretical Issues in Ergonomics Science, 5(2), 113-153.

Klein, G. (2000). Analysis of Situation Awareness from Critical Incident Reports. In D. J. Garland (Ed.), Situation Awareness Analysis and Measurement (pp. 51-71). Mahwah, NJ: Lawrence Erlbaum Associates.

Miller, C. A., Hannen, M., \& Guerlain, S. (1999). The rotorcraft pilot's associate cockpit information manager: acceptable behavior from a new crew member? Paper presented at the American Helicopter Society 55th Annual Forum.

Miyata, Y., \& Norman, D. A. (1986). Psychological issues in support of multiple activities. In D. A. Norman \& S. W. Draper (Eds.), User Centered System Design: New Perspectives on Human Computer Interaction (pp. 265-284). Hillsdale, NJ: Lawrence Erlbaum.

Norman, D. A. (1986). Cognitive Engineering. In D. A. Norman \& S. W. Draper (Eds.), User Centered System Design: New Perspectives on Human Computer Interaction (pp. 31-61). Hillsdale, NJ: Lawrence Erlbaum.

NTSB. (1988). Aircraft Accident Report, Technical Report NTSB-AAR-88-05: National Transportation Safety Board.

Olson, G. M., \& Olson, J. S. (2003). Groupware and Computer-Supported Cooperative Work. In J. A. Jacko \& A. Sears (Eds.), The Human-Computer Interaction Handbook. Mahwah, NJ: Lawrence Erlbaum.

Parasuraman, R., Mouloua, M., Molloy, R., \& Hilburn, B. (1996). Monitoring of automated systems. In R. Parasuraman \& M. Mouloua (Eds.), Automation and human performance: Theory and applications (pp. 91-115).

Parasuraman, R., \& Riley, V. (1997). Humans and Automation: Use, Misuse, Disuse, Abuse. Human Factors, 39(2), 230-253.

Parasuraman, R., Sheridan, T. B., \& Wickens, C. D. (2000). A Model for Types and Levels of Human Interaction with Automation. IEEE Transactions on Systems, Man, and Cybernetics, 30(3), 286-297.

Rodgers, M. D., Mogford, R. H., \& Strauch, B. (2000). Post Hoc Assessment of Situation Awareness in Air Traffic Control Incidents and Major Aircraft Accidents. In D. J. 
Garland (Ed.), Situation Awareness Analysis and Measurement (pp. 73-112).

Mahwah, NJ: Lawrence Erlbaum Associates.

Ruff, H. A., Narayanan, S., \& Draper, M. H. (2002). Human Interaction with Levels of Automation and Decision-Aid Fidelity in the Supervisory Control of Multiple Simulated Unmanned Air Vehicles. Presence, 11(4), 335-351.

Santoro, T. P., \& Amerson, T. L. (1998). Definition and Measurement of Situation Awareness in the Submarine Attack Center. Paper presented at the Command and Control Research and Technology Symposium.

Scallen, S. F., \& Hancock, P. A. (2001). Implementing Adaptive Function Allocation. International Journal of Aviation Psychology, 11(2), 197-221.

Scerbo, M. (1996). Theoretical perspectives on adaptive automation. In R. Parasuraman $\&$ M. Mouloua (Eds.), Automation and human performance: Theory and applications (pp. 37-63).

Shingledecker, C. A. (1987). In-flight workload assessment using embedded secondary radio communication tasks (282). Neuilly Sur Seine, France: Advisory group for Aerospace research and Development.

Tang, J. C., Yankelovich, N., \& Begole, J. (2000). ConNexus: Instant Messaging for the Workplace: Sun Microsystems.

Taylor, R. M., Bonner, M. C., Dickson, B., Howells, H., Miller, C. A., Milton, N., Pleydell-Pearce, K., Shadbolt, N., Tennison, J., \& Whitecross, S. (2002). Cognitive Cockpit Engineering: Coupling Functional State Assessment, Task Knowledge Management, and Decision Support for Context-Sensitive Aiding. In M. McNeese \& M. A. Vidulich (Eds.), Cognitive Systems Engineering in Military Aviation Environments: Avoiding Cogminutia Fragmentosa!: A report produced under the auspices of The Technical Cooperation Programme Technical Panel HUM TP-7 Human Factors in Aircraft Environments (HSIAC-SOAR-2002-01) (pp. 253-312). Wright Patterson Air Force Base: Human Systems Information Analysis Center.

Taylor, R. M., Howells, H., \& Watson, D. (2000). The cognitive cockpit: operational requirement and technical challenge. In P. T. McCabe \& M. A. Hanson \& S. A. Robertson (Eds.), Contemporary Ergonomics 2000 (pp. 55-59). London: Taylor and Francis

Thorsberg, F. (2003, April 17). Can Instant Messaging Really Be Safe? PCWorld. Retrieved December 11, 2003, from the World Wide Web: http://www.pcworld.com/news/article/0,aid,110301,00.asp 
Tsang, P., \& Wilson, G. F. (1997). Mental Workload. In G. Salvendy (Ed.), Handbook of Human Factors and Ergonomics (2nd ed., pp. 417-489). New York: John Wiley $\&$ Sons, Inc.

Wickens, C. D. (1995). Situation awareness: Impact of automation and display technology. Paper presented at the Situation Awareness: Limitations and Enhancements in the Aviation Environment, Neuilly Sur Seine, France.

Wickens, C. D., \& Hollands, J. G. (2000). Engineering Psychology and Human Performance (Third ed.). Upper Saddle River, NJ: Prentice-Hall Inc.

Williges, R. C., \& Wierwille, W. W. (1979). Behavioral Measures of Aircrew Mental Workload. Human Factors, 21(5), 549-574.

Wilson, G. F., \& Russell, C. A. (2004). Psychophysiologically Determined Adaptive Aiding in a Simulated UCAV Task. Paper presented at the Human Performance, Situation Awareness and Automation Technology II Conference, Daytona Beach, FL. 